\title{
Determinação do sexo em catfish de canal (Ictalurus punctatus) utilizando endoscopia
}

\author{
Fernando Souza Mendonça De Lima ${ }^{1}$, Luis David Solis Murgas², Viviane De Oliveira Felizardo ${ }^{3}$, Adriano \\ Carvalho Costa ${ }^{4}$, Bruno Olivetti De Mattos ${ }^{4}$, Daniela Aparecida De Jesus Paula ${ }^{5}$
}

\section{RESUMO}

A identificação precoce do sexo em peixes é um procedimento importante para o desenvolvimento de estratégias que facilitem o manejo no plantel de reprodutores. A endoscopia pode ser utilizada para essa identificação, embora em peixes seu uso ainda seja restrito. Diante disso, o objetivo deste trabalho foi validar o uso do endoscópio rígido via poro urogenital para determinação do sexo do catfish de canal (Ictalurus punctatus). O equipamento utilizado foi um endoscópio Multipurpose Rigid ${ }^{\mathrm{TM}} 30^{\circ} \mathrm{com} 2,7 \mathrm{~mm}$ de diâmetro e $18 \mathrm{~cm}$ de comprimento. Foram examinados 60 peixes com peso variando entre 86 e 1.032 gramas nos quais foi introduzido o endoscópio via poro genital e, logo em seguida, eutanaziados para confirmação do sexo. Amostras de tecido gonadal foram coletadas para avaliação histológica do estádio de maturação e confirmação do sexo. Os resultados mostraram que dos 60 peixes examinados, 57 tiveram confirmação positiva do sexo via endoscopia, o que corresponde a 95\% da amostra. A utilização da endoscopia para determinação do sexo em I. punctatus via poro genital é viável.

Palavras-chave: Peixe, gônadas, sexagem.

\section{ABSTRACT}

\section{Sex determination in channel catfish (Ictalurus punctatus) using endoscopy}

Sex identification in fish is an important practice for the development of broodstock management strategies. Endoscopy can be used for sex identification, although in fish, it is still restricted. Therefore, the objective of this study was to validate the use of rigid endoscope via the urogenital pore to determine the sex of the catfish (Ictalurus punctatus afinesque, 1818). The equipment used was a Multi-Purpose Rigid TM $30^{\circ}$ endoscope, with 2.7 mm in diameter and $18 \mathrm{~cm}$ in length. We examined 60 fish weighing between 86 and 1032 grams, in which the endoscope was introduced via the genital pore and then immediately euthanized to confirm the sex. Gonadal tissue samples were collected for histological assessment of maturation stage and sex confirmation. The results showed that of the 60 fish examined, 57 had positive sex confirmation by endoscopy, corresponding to $95 \%$ of the sample. The results show that it is feasible the use of endoscopy for sex determination in catfish via genital pore.

Key words: Fish, gonads, sexing.

\footnotetext{
Recebido para publicação em novembro de 2010 e aprovado em abril de 2011

${ }^{1}$ Médico Veterinário. Departamento de Medicina Veterinária, Universidade Federal de Lavras, caixa postal: 3037, Centro, 37200-000, Lavras, Minas Gerais, Brasil. pdminas@yahoo.com.br

${ }^{2}$ Médico Veterinário, Doutor. Departamento de Medicina Veterinária, Universidade Federal de Lavras, caixa postal: 3037, Centro, 37200-000, Lavras, Minas Gerais, Brasil. lsmurgas@ufla.br Autor para correspondência

${ }^{3}$ Bióloga, Doutora. Departamento de Medicina Veterinária, Universidade Federal de Lavras, caixa postal: 3037, Centro, 37200-000, Lavras, Minas Gerais, Brasil. viviofbio@yahoo.com.br.

${ }^{4}$ Zootecnista. Departamento de Zootecnia, Universidade Federal de Lavras, caixa postal: 3037, Centro, 37200-000, Lavras, Minas Gerais, Brasil. acarvalhocosta@gmail.com. Z Zootecnista, Mestre. Departamento de Zootecnia, Universidade Federal de Lavras, caixa postal: 3037, Centro, 37200-000, Lavras, Minas Gerais, Brasil. daniufla2002@yahoo.com.br.
} 


\section{INTRODUÇÃO}

O Ictalurus punctatus ou catfish de canal é um peixe da ordem Siluriformes, originário dos Estados Unidos, encontrado nos Estados do Golfo do México e do Vale do Mississipi. Encontra-se introduzido em províncias do Canadá e em todos os Estados Unidos, assim como em muitos outros países do mundo (Wellborn, 1988). O I. punctatus possui reprodução anual e o desenvolvimento ovariano é sincronizado, ocorrendo um evento ovulatório todos os anos (Silverstein \& Small, 2004).

Segundo Furuya \& Ribeiro (1998), o catfish de canal apresenta uma série de caracteres desejáveis para o cultivo, como: não se reproduz nos viveiros, apresenta facilidades em liberar a desova pelo processo artificial, aceita facilmente alimentação artificial, adapta-se a variações bruscas de temperatura e aos vários sistemas de cultivo empregados na sua produção, dentre outros.

A reprodução desse peixe em cativeiro necessita da indução hormonal, como nas espécies de piracema brasileiras. Entretanto, o sucesso dessa técnica é dependente do hormônio utilizado e da espécie, mas, principalmente, da determinação exata do momento do início do protocolo de indução, o qual depende do estádio de maturação gonadal (Muniz et al., 2008).

A identificação precoce do sexo em peixes é um procedimento importante para o desenvolvimento de estratégias que facilitem o manejo do plantel de reprodutores. Aliado a essa sexagem precoce, a visualização das gônadas para determinar a maturação gonadal é de suma importância para o início dos protocolos de indução hormonal (Hurvitz et al., 2007).

Atualmente nas pisciculturas, a principal técnica de sexagem e determinação do estágio de maturação gonadal empregada na reprodução é baseada na visualização de características externas como aumento do volume do abdômen, hiperemia e dilatação do poro urogenital (Felizardo et al., 2010). Tais técnicas, por serem subjetivas, podem variar de acordo com as características de cada espécie ou mesmo de indivíduo, resultando muitas vezes em baixa eficiência reprodutiva nas pisciculturas. Assim, técnicas que possibilitem a diferenciação sexual precoce e a indicação da maturação gonadal dos reprodutores podem propiciar aos produtores melhores técnicas de manejo para os diferentes sexos, podendo assim levar a uma economia de tempo e dinheiro na produção de alevinos (Goos, 1993).

O endoscópio tem sido uma ferramenta útil na determinação do sexo e da maturação gonadal de esturjões (Acipenser oxyrinchus (Hernandez-Divers et al., 2004; Hurvitz et al., 2007), da truta small brook Salvelinus fontinalis (Swenson et al., 2007) e em duas espécies de peixes nativos reofílicos, a curimba Prochilodus spp. e o surubim Pseudoplatystoma coruscans (Kynard \& Kieffer, 2002).
Apesar da endoscopia não ser uma técnica nova em peixes, seu uso ainda é muito restrito no Brasil, mas essa técnica vem sendo bastante utilizada em vários países do mundo (Hurvitz et al., 2007). A técnica apresenta algumas características que o torna confiável para utilização na sexagem, como: (1) não causar lesão às estruturas reprodutivas dos peixes; (2) utilização em campo em alguns minutos; (3) utilização em qualquer condição de tempo; (4) possibilidade de distinguir oócitos que irão ficar maduros na próxima estação reprodutiva; (5) pode ser facilmente ensinada a técnicos de campo dentre outras (Kynard \& Kieffer, 2002).

Diante disso, o objetivo deste trabalho foi validar o uso do endoscópio rígido para determinação do sexo de $I$. punctatus através do poro urogenital.

\section{MATERIAL E MÉTODOS}

Este trabalho foi realizado na estação de piscicultura da Universidade Federal de Lavras - UFLA, no mês de maio de 2009. Foram utilizados 60 exemplares de $I$. punctatus com peso que variou de 86 a 1.032 gramas, para verificar se a metodologia pode ser empregada em peixes de diferentes tamanhos. Os peixes estavam acondicionados em tanque-rede dentro de um tanque escavado de 40 $\mathrm{m}^{2}$ e recebiam ração comercial ad libitum.

Para a realização das avaliações, cada peixe foi retirado do tanque-rede e posteriormente colocado em balde para imediatamente ser conduzido até a sala de necrópsia da estação de piscicultura, onde eles foram anestesiados com óleo de cravo (20 mg/L) e em seguida foi introduzido o endoscópio via poro genital para visualização das gônadas via imagem no computador.

O equipamento utilizado foi um endoscópio rígido Karl Storz Multipurpose Rigid ${ }^{\mathrm{TM}} 30^{\circ} \mathrm{com} 2,7$ mm de diâmetro e $18 \mathrm{~cm}$ de comprimento, acoplado a uma camisa endoscópica de $5 \mathrm{~mm}$ para insuflação de soro fisiológico estéril, além de cabo de fibra ótica e fonte de luz alógena de 150 Watts. Para visualização e gravação das imagens no notebook, foi acoplado ao endoscópio uma microcâmera MedCam ligada a uma placa de captura de imagens Dazzle Pinnacle ${ }^{\mathrm{TM}}$.

A técnica consistiu em identificar as gônadas e suas características, como coloração, tamanho e vascularização. As fêmeas foram caracterizadas com a visualização de oócitos, sendo então as gônadas classificadas como imaturas ou maduras, de acordo com Vazzoler (1996), já a caracterização dos machos era feita pela visualização das projeções digitiformes dos testículos através da parede translúcida do canal espermático.

Após o exame endoscópico, os peixes foram insensibilizados com gelo e logo após eutanaziados para dissecação. Todos os peixes foram pesados ( $\mathrm{g}$ ) com balança

Rev. Ceres, Viçosa, v. 58, n.3, p. 284-287, mai/jun, 2011 
eletrônica e feita a biometria para comprimento total $(\mathrm{cm})$ e comprimento padrão $(\mathrm{cm})$. Na dissecação, foi realizada a visualização das gônadas para comparar com as imagens geradas pelo endoscópio. Amostras de tecido gonadal foram coletadas para posterior confirmação dos sexos dos animais e estádio de maturação.

Para confirmação do sexo e estádio de maturação, as amostras das gônadas de cada peixe foram fixadas em Solução de Bouin por 12 a 14 horas, e logo depois armazenadas em álcool $70 \%$ para posterior processamento pelas técnicas histológicas de rotina no Departamento de Patologia Veterinária da UFLA. O estudo histológico das lâminas foi realizado em microscópio óptico com aumento de 400 vezes.

Os estádios de maturação foram determinados de acordo com as características histológicas das gônadas, seguindo a escala de desenvolvimento gonadal estabelecida por Bazzoli (2003): repouso, maturação inicial, maturação avançada/maduro e desovado/espermiado.

\section{RESULTADOS E DISCUSSÃO}

Por meio da imagem gerada das gônadas, pelo exame endoscópico, foi possível identificar no interior do ovário das fêmeas a presença de oócitos e suas características, como cor, tamanho e ausência de vascularização. Já nos machos, a visualização das projeções digitiformes era realizada por meio da parede translúcida do canal espermático, pois esta espécie possui testículos do tipo franjado, não possibilitando a inserção mais profunda do endoscópio.

Foi possível o exame com a utilização do endoscópico nas fêmeas, independentemente do tamanho, mas nos machos, principalmente os que se encontravam com menos de $30 \mathrm{~cm}$, a introdução do endoscópio só foi possível sem a utilização da camisa endoscópica, o que tornava a visualização mais difícil, pois não se podia fazer a insuflação com soro fisiológico estéril. Ortenburger et al. (1996), realizando a sexagem de Arctic charr, observaram resultados semelhante ao descrito neste trabalho.

Dos 60 peixes examinados utilizando a endoscopia via poro urogenital e confirmado via histologia das gônadas, foi observada correta classificação do sexo em 95\% (n=57) dos animais (Tabela 1), resultado semelhante, utilizando a mesma técnica, foi encontrado em outras espécies de peixes por Ortenburger et al. (1996) e Kynard \& Kieffer (2002).

Tabela 1. Acurácia da endoscopia para sexagem do I. punctatus comparado com corte histológico das gônadas para confirmação do sexo

\begin{tabular}{lcc}
\hline Sexo & Endoscopia & Avaliação histológica \\
\hline Macho & 22 & 25 \\
Fêmea & 35 & 35 \\
Indeterminado & 3 & 0 \\
\hline Total & $\mathbf{6 0}$ & $\mathbf{6 0}$ \\
\hline
\end{tabular}

A não identificação do sexo em três peixes machos pode ser atribuída ao pequeno tamanho dos exemplares, sendo classificados como indeterminado. Em seu trabalho, Ortenburger et al. (1996) atribuíram para a não identificação do sexo em alguns peixes o fato de ocorrer ruptura acidental do ducto espermático em alguns machos após a inserção do endoscópio, tal situação poderia ter ocorrido neste estudo.

Alves \& Sawaya (1975) afirmaram que o estádio de desenvolvimento gonadal imaturo pode apresentar macroscopicamente gônadas indiferenciadas, enquanto Agostinho et al. (1982) informaram serem as gônadas masculinas e femininas perfeitamente distinguíveis a olho nu em todos os estádios de desenvolvimento. Como neste trabalho todas as fêmeas encontravam-se em estádio avançado de maturação gonadal e os machos encontravam-se espermiados, seria necessário mais estudos utilizando essa técnica em outras fazes do ciclo reprodutivo do I. puntatus para determinar se seria possível a utilização dessa técnica para sexar animais imaturos.

No exame histológico dos machos do I. punctatus, os testículos apresentavam-se revestidos pela túnica albugínea, camada de tecido conjuntivo fibroso que emite septos para o seu interior, separando e sustentando os túbulos seminíferos, e todas as lâminas apresentavamse com lume aberto e presença, em algumas, de espermatozoides residuais e parede constituída de espermatogônias, característico de testículos espermiados, segundo Bazzoli (2003).

Já no exame histológico das fêmeas do I. punctatus os ovários apresentavam-se revestidos pela túnica albugínea formando lamelas ovulígeras, tendo em todas as lâminas sido observado a presença de oócitos com glóbulos de vitelo ocupando quase todo o citoplasma e com reduzido número de oócitos pré-vitelogênicos, o que caracteriza fêmeas em estado de maturação avançada, segundo Bazzoli (2003).

Para caracterizar as fêmeas, pelas imagens geradas após a inserção do endoscópio pelo poro urogenital pôde se observar numerosas estruturas cilíndricas de coloração amarelo-viva sem a presença de vascularização; portanto, caracterizados como oócitos maduros (Figura 1). Já para os machos, pôde se visualizar, por meio da parede translúcida do ducto espermático, projeções digitiformes de coloração esbranquiçada, caracterizando-os apenas como machos (Figura 2).

Dos 60 peixes examinados, 25 foram machos e 35 fêmeas, todos confirmados via visualização de corte histológico das gônadas. Entre os machos o menor apresentou comprimento total de 22,2 e o maior, $44,7 \mathrm{~cm}$, e o peso variou de 108 a $1.032 \mathrm{~g}$. Entre as fêmeas examinadas, a menor apresentou comprimento total de 20 e a maior, 40,4 $\mathrm{cm}$, e o pesou variou de 86 a 810 g. Em relação ao estágio

Rev. Ceres, Viçosa, v. 58, n.3, p. 284-287, mai/jun, 2011 

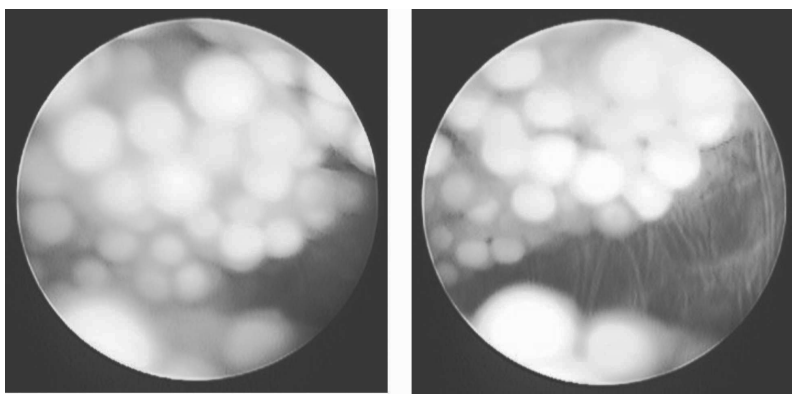

Figura 1. Imagem gerada pela endoscopia da fêmea na qual se pode observar oócitos e a ausência de vascularização.

de maturação das gônadas, todas as fêmeas encontravam-se em estádio avançado, enquanto todos os machos, em estádio espermiado.

Os testículos do I. punctatus são órgãos pares e apresentaram projeções digitiformes ou franjadas, nas quais se comunicavam com os ductos espermáticos; ainda foi observado uma divisão em duas partes distintas em cor, morfologia e textura tecidual, sendo a parte anterior espermatogênica e a posterior glandular. De acordo com Sneed \& Howard (1963), a parte anterior epsermatogênica é branca com lóbulos largos e consiste em tecido muito macio, enquanto a posterior glandular é rósea com os lóbulos muito finos e o tecido mais duro.

O ovário de I. punctatus, igualmente como descrito por Silverstein \& Small (2004), é oco e o lúmen conecta-se ao oviduto. Os ovários, como os testículos, estão anatomicamente relacionados cranialmente com a bexiga gasosa e caudalmente com os rins, além de unirem-se ao rim e à bexiga gasosa por tecidos mesentéricos.

\section{CONCLUSÂO}

Com as análises realizadas, pode-se concluir que tanto o equipamento quanto a técnica empregada mostraram-se eficientes para visualização das gônadas e determinação do sexo, e sua classificação quanto ao estágio de maturação gonadal em fêmeas maduras, podendo ser potente ferramenta na seleção de reprodutores dessa espécie, aptos a receberem a indução hormonal.

\section{REFERÊNCIAS BIBLIOGRÁFICAS}

Agostinho AA, Narahara MY \& Godinho HM (1982) Morfologia dos ovários de Plecostomus commersonii (Valenciennes, 1840) Osteichthyes - Loricariidae: desenvolvimento dos ovócitos e escala de maturidade. Revista Brasileira de Biologia, 42:71-77.

Alves MJM \& Sawaya P (1975) Sobre a reprodução da Sardinhabandeira, Opisthonema oglinum (Le Seuer) na costa do estado do Ceará (Brasil). Arquivo de Ciência do Mar, 15:19-28.

Bazzoli N (2003) Parâmetros reprodutivos de peixes de interesse comercial na região de Pirapora: águas, peixes e pescadores do São Francisco das Minas Gerais. Belo Horizonte: PUC Minas/ CNPq-PADCT. p.291-306.
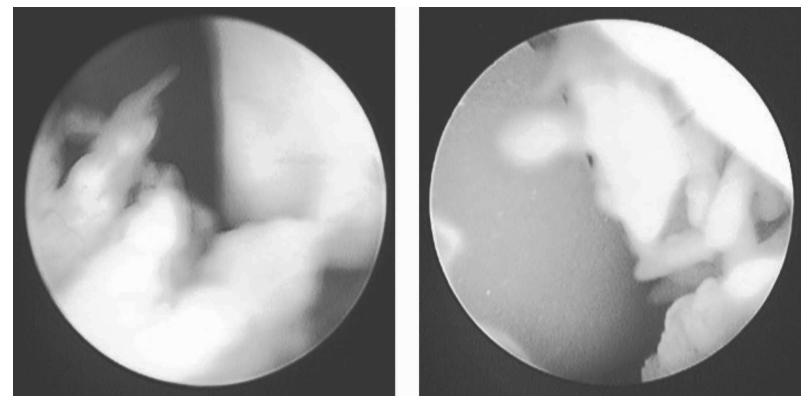

Figura 2. Imagem gerada pela endoscopia do macho na qual observam-se as projeções digitiformes ou franjas dos testículos.

Felizardo VO, Mello RA, Murgas LDS, Andrade ES, Drumond MM \& Rosa PV (2010) Effect of cryopreservant combinations on the motility and morphology of curimba (Prochilodus lineatus) sperm. Animal Reproduction Scienc, in press.

Furuya WM \& Ribeiro RP (1998) Criação de espécies nativas e criação de espécies exóticas. Maringá, FADEC-UEM. 92p.

Goos HJT (1993) Pubertal development: big questions, small answers. In: Goos HJT (ed.) Cellular Communication in Reproduction. Facchinetti. p11-20.

Hernandez-Divers SJ, Bakal RS, Hickson BH, Rawlings CA, Wilson HG \& Radlinsky MA (2004) Endoscopic sex determination and gonadal manipulation in Gulf of Mexico sturgeon (Acipenser oxyrinchus desotoi). Journal of Zoology, 35:459-470.

Hurvitz A, Jackson K, Degani G \& Levavi-Sivan B (2007) Use of endoscopy for gender and ovarian stage determinations in Russian sturgeon (Acipenser gueldenstaedtii) grown in aquaculture. Aquaculture, 270:158-166.

Kynard B \& Kieffer M (2002) Use of a borescope to determine the sex and egg maturity stage of sturgeons and the effect of borescope use on reproductive structures. Journal of Applied Ichthyology, 18:505-508.

Muniz JASM, Catanho MTJA \& Santos AJG (2008) Influência do fotoperíodo natural na reprodução induzida do tambaqui, Colossoma macropomum (CUVIER, 1818). Boletim do Instituto de Pesca, 34:205-211.

Ortenburger AI, Jansen ME \& Whyte SK (1996) Nonsurgical videolaproscopy for determination of reproductive status of the Arctic charr. Canadian Veterinary Journal, 37:96-100.

Silvertein J \& Small BC (2004) Reproductive and Culture of Channel Catfsih. Elsevier.

Sneed KE \& HOWARD PC (1963) The morphology of the testes and accessory reproductive glands of the catfishes (Ictaluridae). Copeia, 4:606-611.

Swenson EA, Amanda E, Rosenberger AE \& Howell PJ (2007) Validation of endoscopy for determination of maturity in small salmonids and sex of mature individuals. Transactions of the American Fisheries Society, 4:994-998.

Vazzoler AEM (1996) Biologia da reprodução de peixes teleósteos: teoria e prática. Maringá, EDUEM/Nupélia. 169p.

Wellborn TL (1999) Channel Catfish: life history and biology. Nova Orleans: Southern Regional Aquaculture Center (SRAC), Texas Agricultural Extension Service, University of Florida. Capturado em 13 de Dezembro. Online. Disponível em: http:// www.farminfo.org/aquaculture/chancat.htm Acessado em: 04 de fevereiro de 2010. 